\title{
Interferometric phase-dispersion microscopy
}

\author{
Changhuei Yang, Adam Wax, Irene Georgakoudi, and Eugene B. Hanlon \\ George Harrison Spectroscopy Laboratory, Massachusetts Institute of Technology, Cambridge, Massachusetts 02139
}

Kamran Badizadegan

Department of Pathology, Children's Hospital, Boston, Massachusetts 02115

Ramachandra R. Dasari and Michael S. Feld

George Harrison Spectroscopy Laboratory, Massachusetts Institute of Technology, Cambridge, Massachusetts 02139

Received July 20, 2000

\begin{abstract}
We describe a new scanning microscopy technique, phase-dispersion microscopy (PDM). The technique is based on measuring the phase difference between the fundamental and the second-harmonic light in a novel interferometer. PDM is highly sensitive to subtle refractive-index differences that are due to dispersion (differential optical path sensitivity, $5 \mathrm{~nm}$ ). We apply PDM to measure minute amounts of DNA in solution and to study biological tissue sections. We demonstrate that PDM performs better than conventional phasecontrast microscopy in imaging dispersive and weakly scattering samples. (C) 2000 Optical Society of America OCIS codes: $\quad 040.2840,110.0180,110.4500,120.5050,170.3880$.
\end{abstract}

Conventional phase-contrast microscopy (PCM) forms images by phase shifting the light field scattered from a specimen and interfering it with the unscattered light field. PCM is able to render subcellular structures visible without staining. However, it can be applied only to specimens that scatter a significant amount of light, and the information it provides is largely qualitative. Various interference microscopes have been developed that complement PCM with their ability to image weakly scattering specimens and provide quantitative information. However, these methods are difficult to use and they contain inherent artifacts, which have likely prevented them from supplanting PCM. ${ }^{1}$

The nature of PCM and interference microscopy limits their applications to two-dimensional (2D) imaging of thin samples. Low-coherence interferometry techniques, such as optical coherence tomography, ${ }^{2}$ open exciting possibilities of using phase-based measurements for three-dimensional (3D) microscopic imaging. Several research groups have already applied phase-sensitive interferometric techniques to provide high-resolution Doppler optical coherence tomography images, ${ }^{3}$ to reduce interferometer jitter, ${ }^{4}$ and to compare phase differences at adjacent points on a target. ${ }^{5}$ The last of these is an extension of Nomarski interference microscopy ${ }^{1}$ to $3 \mathrm{D}$ imaging.

We present a new and very sensitive microscopy imaging technique, phase-dispersion microscopy (PDM). This technique is based on measuring the phase difference between the fundamental and the second-harmonic unscattered light transmitted through a sample. PDM employs a novel interferometer that eliminates all noise that is due to optical path-length fluctuations. ${ }^{7}$ In other phasemeasurement techniques, ${ }^{5,6}$ it is difficult to account for minute interferometer path-length differences in the measured phase. Thus, without an independent way of measuring these differences and eliminating the associated motional jitter, phase measurements cannot directly yield physically relevant information. In contrast, the phase measured in our new technique is independent of path-length errors. As an initial demonstration we have used the new technique to measure very small anomalous phase-velocity differences experienced by ballistic light during propagation through turbid media. ${ }^{7}$

In this Letter we demonstrate the application of this phase-measurement method to 2D PDM imaging. The capabilities of this system are compared with those of conventional PCM, the current standard. We first show that this new method can provide quantitative information by measuring the refractive-index dispersion of very dilute DNA-water solutions. We then demonstrate the sensitivity of the technique and its image formation capabilities by applying it to an unstained tissue section. Finally, we discuss the extension of this technique to 3D imaging.

PDM is based on a modified low-coherence Michelson interferometer (Fig. 1). The input light is created by overlapping beams of laser light at the fundamental and the second-harmonic frequencies, which form a two-color composite beam. The source is a lowcoherence Ti:sapphire laser that produces 150-fs pulses at $800 \mathrm{~nm}$ with a beam diameter of $2.1 \mathrm{~mm}$ (FWHM) at the input to the interferometer, and the second harmonic, with a beam diameter of $1.1 \mathrm{~mm}$, is generated by a standard frequency doubler. The composite beam is split in two at the beam splitter. One part is focused on the target sample of known thickness and makes two passes through it, while the other passes through a compensator in a similar fashion. The powers of the 400- and 800-nm components at the sample are 7.0 and $2.8 \mathrm{~mW}$, respectively. Achromatic $10 \times$ microscope objectives focus the composite beam onto the sample with a FWHM of $\sim 7 \mu \mathrm{m}$ at both wavelengths; however, difficulty in aligning the returning path to overlap with the incoming path degrades the resolution to $\sim 10 \mu \mathrm{m}$. Note that finer resolution is achievable by use of higher-power objectives and improved alignment.

The reference mirror moves at a constant velocity of $1 \mathrm{~mm} / \mathrm{s}$ and induces a Doppler shift in the returning 


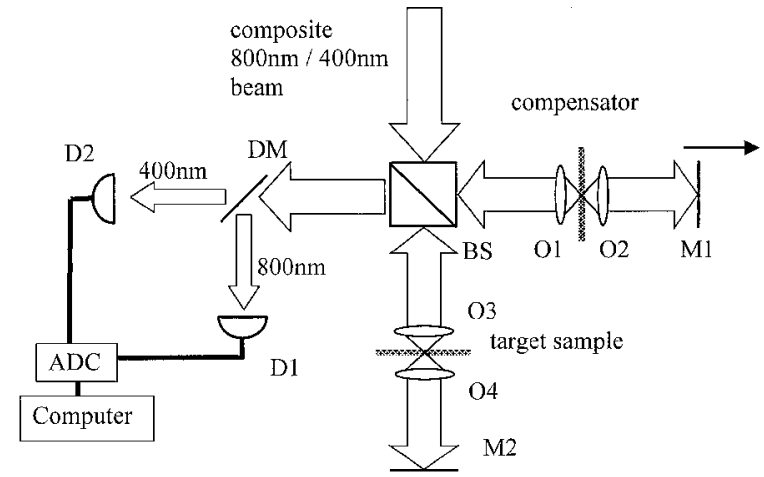

Fig. 1. Experimental setup: M1, M2, mirrors (M1 is the moving reference mirror); BS, beam splitter; O1-O4, microscope objectives; D1, D2, photodetectors; DM, 400-800-nm dichroic mirror; ADC, analog-digital converter.

beam. The two composite beams then are recombined, separated by their wavelength components with a dichroic mirror, and measured separately by photodetectors. The resulting heterodyne signals at both wavelengths are measured and digitized by a 16-bit $100-\mathrm{kHz}$ analog-digital converter. Each digitized signal is bandpassed around its center heterodyne frequency, as given by the Doppler shift. The filtered signals are then Hilbert transformed, and their respective phases, $\Psi_{1}$ and $\Psi_{2}$, are extracted. ${ }^{3,8}$ The acquisition and processing time for each pixel is $\sim 3 \mathrm{~s}$. Related phase techniques have been used to measure the dispersion property of metals ${ }^{9}$ and the refractive index of air. ${ }^{10}$

It can be seen that a jitter of magnitude $\Delta x$ in either the signal- or the reference-arm length will vary phases $\Psi_{1}$ and $\Psi_{2}$ by $k_{1} \Delta x$ and $k_{2} \Delta x$, respectively, where $k_{1}\left(k_{2}\right)$ is the free-space wave number of the fundamental (second-harmonic) light. As $k_{2}$ is exactly double $k_{1}$, we can totally eliminate the effect of this jitter by subtracting twice $\Psi_{1}$ from $\Psi_{2}$. Note that such elimination is possible only when one wavelength is an integer multiple of the other. This operation yields $\Delta \mathrm{OL}_{k_{2}, k_{1}}$, the optical path-length difference experienced by the two wavelengths in the interferometer, with great sensitivity:

$$
\Delta \mathrm{OL}_{k_{2}, k_{1}}=\left(\Psi_{2}-2 \Psi_{1}\right) / k_{2} .
$$

The sensitivity achieved is $\sim 5 \mathrm{~nm}$ in optical pathlength difference or, equivalently, approximately $9 \times 10^{-2} \mathrm{rad}$ for phase difference with respect to the second-harmonic light. Note that phase measurements are inherently limited to modulus $2 \pi$; therefore measurements of longer path differences require appropriate phase-unwrapping techniques such as those described in Ref. 11. For clarity, we shall limit the present discussion to samples that are sufficiently thin or dilute that phase unwrapping is not required.

By independent measurement of sample thickness $L$, we can evaluate the refractive-index dispersion relative to that of the compensator medium $\left(\Delta n_{400 \mathrm{~nm}}-\right.$ $\Delta n_{800 \mathrm{~nm}}$ ) between the wavelengths by use of

$$
\Delta n_{400 \mathrm{~nm}}-\Delta n_{800 \mathrm{~nm}}=\Delta \mathrm{OL}_{k_{2}, k_{1}} / L,
$$

where $\Delta n_{400 \mathrm{~nm}}\left(\Delta n_{800 \mathrm{~nm}}\right)$ is the difference in refractive index between the sample and the compensator medium at the wavelength $400 \mathrm{~nm}(800 \mathrm{~nm})$. The sensitivity of our system permits us to detect refractive-index dispersion as small as $5 \times 10^{-6}$ for a 1-mm sample. This sensitivity improves for thicker samples.

To demonstrate the sensitivity of this method we measure the refractive-index dispersion change produced by addition of a small amount of DNA to water. The experiment is performed by replacement of the microscope objectives (O1 and O2), and the target sample with a cuvette of very dilute herring tests DNA $(0.014 \%$ vol. concentration). The cuvette is $10 \mathrm{~mm}$ thick, which makes $L=20 \mathrm{~mm}$ because of the system's double-pass configuration. The compensator and its associated objectives ( $\mathrm{O} 3$ and $\mathrm{O} 4$ ) are correspondingly replaced by a cuvette containing only water. The measured refractive-index dispersion, based on ten separate measurements, is $(2.27 \pm 0.04) \times 10^{-6}$.

We can draw some distinctions between PDM and PCM. PCM is a qualitative technique, and in the resulting image it is difficult to separate the contributions from absorption and phase shift. PDM provides a quantitative measurement of the phase shift. In addition, whereas PCM relies on small phase shifts between the scattered and unscattered light from the target for contrast, PDM directly measures the small phase shifts of the unscattered light that are associated with the refraction of the target. This sensitivity results from the fact that interference-based techniques detect unscattered light far more efficiently than scattered light. ${ }^{12,13}$ Therefore PDM can render useful phase images when it is used on samples that are weakly scattering or do not scatter at all, whereas PCM has difficulty with such samples.

As an illustration, we compare the performance of PDM and PCM on similarly prepared samples composed of a drop of water and a drop of DNA solution (1.0\% vol. concentration) sandwiched between two coverslips (Fig. 2). The separation between the coverslips is $170 \mu \mathrm{m}$. As is evident from Fig. 2, PDM can easily distinguish between the two drops and provides a refractive-index dispersion value for the DNA solution. In contrast, PCM does not distinguish between the two drops. Interestingly, the refractive-index dispersion measured in this experiment, $(1.3 \pm 0.2) \times 10^{-4}$, differs from the value $1.6 \times 10^{-4}$ extrapolated from the cuvette experiment, based only on the ratio of the concentrations. This difference can be attributed to the fact that the refractive index depends on scatterer size as well as concentrations. Thus, at higher concentration, the formation of DNA aggregates, which behave as scatterers, effectively alters the refractive index. In recent work ${ }^{7}$ it was experimentally verified that the refractive index depends strongly on scatterer size.

To demonstrate further the capability of PDM we compare PDM and PCM images of a brain tissue sample. A 16- $\mu \mathrm{m}$-thick sample was prepared from a frozen brain tissue block by use of a microtome. The sample was obtained from the autopsy material of an Alzhemier's disease patient and sandwiched between two coverslips. We applied a drop of glycerol to keep the sample moist and to provide index matching. We 


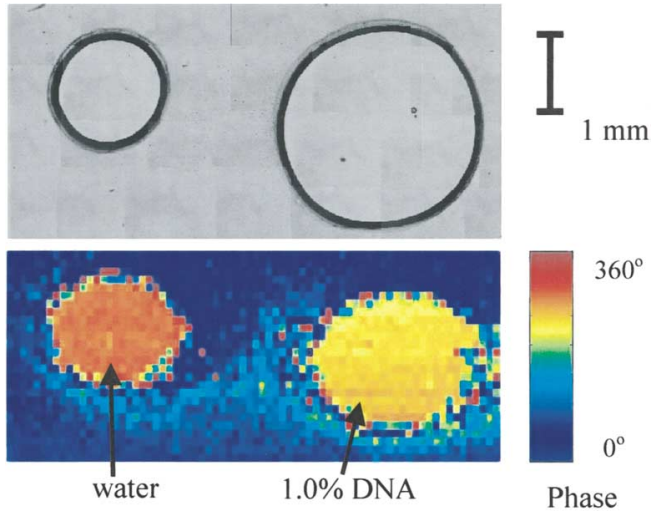

Fig. 2. Comparison of images from (top) PCM and (bottom) PDM of a drop of water and a drop of $1.0 \%$ DNA solution sandwiched between two coverslips. We measure the refractive-index dispersion $\left(\Delta n_{400 \mathrm{~nm}}-\Delta n_{800 \mathrm{~nm}}\right)$ of the DNA solution to be $(1.3 \pm 0.2) \times 10^{-4}$.

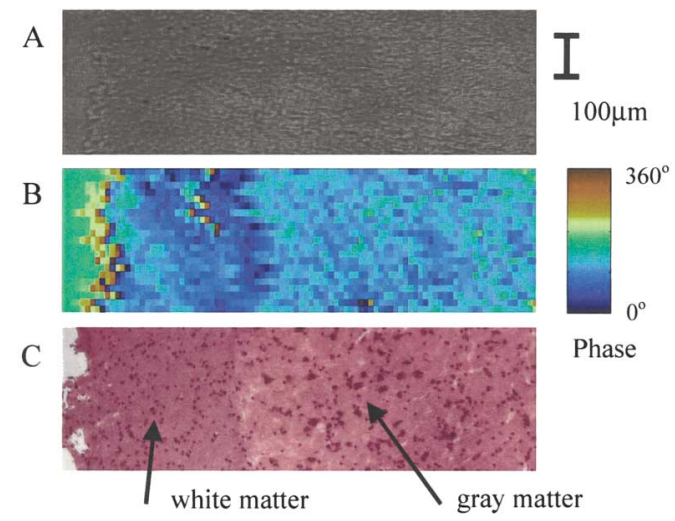

Fig. 3. Images of a white matter-gray matter interface in a 16- $\mu \mathrm{m}$-thick brain sample. A, PCM image; B, PDM image; $\mathrm{C}$, an adjacent frozen section stained with hemotoxylin and eosin.

used a compensator in the reference-arm that was identical to the target sample but with no tissue. Figure 3 shows PCM and PDM images taken from the same sample. For comparison, a stained sample from an adjacent thin section is also shown. As can be seen, the PCM image reveals only a slight distinction between the gray and white matter; this is due to the relatively weak scattering of brain tissue. In comparison, the differences between the two types of matter are quite visible with PDM. This contrast can be attributed to differences in the composition of the two tissue types, which give rise to a small but measurable refractive-index dispersion change.

In this Letter we have demonstrated the use of PDM for $2 \mathrm{D}$ imaging. $\mathrm{PDM}$ is also readily adaptable to $3 \mathrm{D}$ imaging, by use of a backscattering geometry, as is done in optical coherence tomography. ${ }^{2,3}$ This demonstration opens the important possibility of creating tomographic phase-dispersion images of in vivo sites. As discussed above, this technique is very sensitive to small biological differences that manifest themselves as changes in the refractive index. In addition, simultaneous measurement of the amplitude and phase of the heterodyne signal yields the real and imaginary parts of the refractive index, providing a more-comprehensive set of data about the scanned sample.

Further, an earlier study ${ }^{7}$ performed on scatterers with this system showed that the refractive index is strongly dependent on scatterer size, scatterer composition, and probe wavelength. Thus, by spectral scanning of the fundamental and the second-harmonic wavelengths, it should be possible to extract precise scatterer size distributions in polydisperse media. It is important to note that such size characterization can far exceed the actual vortex resolution, as phase-based measurements are very sensitive to the spectral variation of the refractive index with scatterer size. This method should complement related intensity-based techniques ${ }^{14}$ by rendering $3 \mathrm{D}$ functional images of the size distribution and chromatin content of cell nuclei-important indicators of precancerous changes in biological tissues.

This work was carried out at the Laser Biomedical Research Center of the Massachusetts Institute of Technology and was by supported by National Institutes of Health (NIH) grant P41-RR02594, National Science Foundation grant 9708265-CHE, and a grant from Hamamatsu Corporation. Adam Wax and Irene Georgakoudi were supported by NIH National Research Service Awards 1 F32 RR05075-01 and 1 F32 CA80345-01, respectively.

\section{References}

1. K. F. A. Ross, Phase Contrast and Interference Microscopy for Cell Biologists (St. Martin's, New York, 1967).

2. U. Morgner, W. Drexler, F. X. Kartner, X. D. Li, C. Pitris, E. P. Ippen, and J. G. Fujimoto, Opt. Lett. 25, 111 (2000).

3. Y. Zhao, Z. Chen, C. Saxer, S. Xiang, J. F. de Boer, and J. S. Nelson, Opt. Lett. 25, 114 (2000).

4. K. M. Yung, S. L. Lee, and J. M. Schmitt, J. Biomed. Opt. 4, 125 (1999).

5. C. K. Hitzenberger and A. F. Fercher, Opt. Lett. 24, 622 (1999).

6. E. Cuche, F. Bevilacqua, and C. Despeursinge, Opt. Lett. 24, 291 (1999).

7. C. Yang, A. Wax, and M. S. Feld are preparing a manuscript to be called "Measurement of anomalous phase velocity of ballistic light in a random medium using a novel interferometer."

8. A. B. Carlson, Communication Systems, 3rd ed. (McGraw-Hill, New York, 1986).

9. H. Matsumoto, Rev. Sci. Instrum. 65, 2894 (1994).

10. H. Matsumoto and L. Zeng, Opt. Commun. 104, 241 (1994).

11. G. Fornaro, G. Franceschetti, R. Lanari, and E. Sansosti, J. Opt. Soc. Am. A 13, 2355 (1999).

12. C. Yang, K. An, L. T. Perelman, R. R. Dasari, and M. S. Feld, J. Opt. Soc. Am. A 16, 866 (1999).

13. A. Wax and J. E. Thomas, J. Opt. Soc. Am. A 15, 1896 (1998).

14. L. T. Perelman, V. Backman, M. Wallace, G. Zonios, R. Manohanan, A. Nusrat, S. Shields, M. Seiler, C. Lima, T. Hamamo, I. Itzkan, J. Van Dam, J. M. Crawford, and M. S. Feld, Phys. Rev. Lett. 80, 627 (1998). 